\title{
BreastCare
}

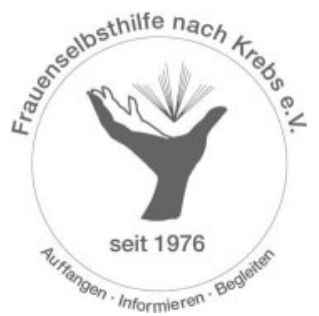

Hilde Schulte

Frauenselbsthilfe nach Krebs, Bundesverband e.V.

Haus der Krebs-Selbsthilfe

Thomas-Mann-Str. 40, 53111 Bonn, Deutschland

Tel. +49 (0)228 33889-404, Fax -401

E-Mail kontakt@frauenselbsthilfe.de

www.frauenselbsthilfe.de

\section{Mammographiescreening - ein wichtiger Baustein der Früherkennung}

\section{Die Vorgeschichte}

Auf Beschluss des Bundesausschusses der Ärzte und Krankenkassen vom 12. September 1996 sollten die Bedingungen für eine Integration des MammographieScreenings in das Krebsfrüherkennungsprogramm der gesetzlichen Krankenversicherung erprobt und festgelegt werden [1]. Der Sachverständigenrat für die konzertierte Aktion im Gesundheitswesen hat in seinem Gutachten 2000/2001 eine Über-, Unter- und Fehlversorgung in der Diagnostik von Brustkrebs festgestellt und zur Vermeidung von Schäden und Kosten die Einführung des Mammographie-Screenings empfohlen. Für die Umsetzung wurde von den Spitzenverbänden der Krankenkassen und der Kassenärztlichen Bundesvereinigung die Planungsstelle Mammographie-Screening eingerichtet. Seit Januar 2004 haben alle Frauen in Deutschland zwischen 50 und 69 Jahren den gesetzlich verbürgten Anspruch auf ein flächendeckendes, qualitätsgesichertes Mammographie-Screening nach europäischen Leitlinien.

\section{Die Chancen}

Die Chancen der Früherkennung durch Screening bestehen darin, eine Erkrankung in einem asymptomatischen Stadium zu entdecken. Das bedeutet: Diagnose zu einem sehr frühen Zeitpunkt, schonendere Operationsverfahren, größere Chancen auf Brusterhaltung (statt
Mastektomie) mit besserem kosmetischen Ergebnis, weniger belastende systemische Therapien und grundsätzlich bessere Heilungschancen. Die Effektivität des Mammographie-Screenings, die Brustkrebssterblichkeit im Altersbereich zwischen 50 und 69 Jahren um etwa 30\% zu senken, wurde in randomisierten Studien bereits in den 1990er Jahren nachgewiesen. Dass mit der Reduzierung der Sterblichkeit eine höhere Lebensqualität einhergeht, versteht sich von selbst.

\section{Die Risiken}

Da sich das Angebot des Screenings an symptomlose, augenscheinlich gesunde Frauen richtet, ist eine besonders sorgfältige Nutzen-Risiko-Abwägung vorzunehmen. Als unerwünschte Nebenwirkung gilt die Strahlenbelastung, der sich $90 \%$ der Screening-Teilnehmerinnen aussetzen, ohne dass sie jemals in ihrem Leben an Brustkrebs erkranken würden. Falsch-positive und falsch-negative Befunde schlagen ebenfalls auf der Schadensseite zu Buche, denn sie führen zu Folgeuntersuchungen und belasten die betroffenen Frauen unnötig.

\section{Irritationen}

Nicht nur die Vor- und Nachteile des MammographieScreenings an sich haben die Gemüter bewegt und er-

\section{KARGER}

Fax +497614520714

E-mail Information@Karger.de www.karger.com/brc www.karger.com

(C) 2007 S. Karger GmbH, Freiburg 


\section{Breast Care}

regt. Die beteiligten Professionen haben sich aus der jeweiligen Interessenlage heraus heftige Diskussionen geliefert und zur Verunsicherung der Frauen beigetragen. Hinzu kommt die unterschiedliche Interpretation von ohnehin widersprüchlichen Daten und die grundsätzlich mangelnde Transparenz in unserem Gesundheitssystem und hier im Speziellen bezüglich Nutzen und Schaden von Früherkennung und Screening. Es sei noch einmal festgehalten: Mammographie-Screening ist eine Ergänzung zu der bisherigen gesetzlichen Früherkennung, kein Ersatz, der Kontakt zum Gynäkologen ist unverändert erforderlich.

\section{Die Modellprojekte}

Drei Modellprojekte wurden eingerichtet: Die Stadt Bremen und die gemischt städtisch/ländliche Region Wiesbaden/Rheingau-Taunus-Kreis haben im Jahr 2001 die Arbeit aufgenommen. Im Jahr 2002 kam als ländlich geprägtes Gebiet mit geringer Bevölkerungsdichte die mobile Mammographie-Einheit Weser-Ems als Modellregion dazu. Alle drei hatten die Aufgabe, die von vielen Experten in Frage gestellte Übertragbarkeit des Mammographie-Screenings nach EU-Leitlinien auf die deutschen Gesundheitsstrukturen zu prüfen. Es ging nicht um den Beweis der inhaltlichen Wirksamkeit des Mammographie-Screenings, der Reduzierung von Sterblichkeit.

\section{Die Ergebnisse}

Der mit Spannung erwartete Abschlussbericht der drei Mammographie-Screening-Modellprojekte ist im Oktober 2006 von der Kooperationsgemeinschaft Mammographie veröffentlicht worden [1]. Die Ergebnisse können sich sehen lassen. Die Brustkrebsentdeckungsrate lag in allen drei Regionen über dem geforderten Mindestwert (7,5 je 1,000 Frauen beim Erstscreening): in Bremen bei 8,7, in Wiesbaden bei 9,4 und in Weser-Ems bei 8,3. Die entdeckten Karzinome liegen erwartungsgemäß vermehrt in den frühen Krankheitsstadien (ohne Lymphknotenbefall), die für die Frauen weniger belastende Therapien und eine bessere Prognose bedeuten. Karzinome unter $10 \mathrm{~mm}$ lagen insgesamt zwischen 25 und $36 \%$ (Vorgabe $>25 \%$ ), Karzinome unter $15 \mathrm{~mm}$ zwischen 58 und 70,3\% (Vorgabe $>50 \%$ ). Auffällig ist der Anteil an in-situ-Karzinomen: Er macht in allen Regionen etwa $20 \%$ aus.

\section{Die Teilnahmequote}

Das Ziel des Screenings, eine Teilnahmequote von 70\%, ist bisher in keinem der bereits eingeführten europäischen Screening-Programme erreicht worden. Eine Teilnahme von $70 \%$ der anspruchsberechtigten Frauen ist erforderlich, damit die Reduzierung der Sterblichkeit von $30 \%$ erreicht werden kann. In den Modellregionen nahmen in der ersten Einladungsrunde 53\% der anspruchsberechtigten Frauen teil, in der zweiten Runde bereits $59 \%$.

\section{Kritiker und Zahlenspiele}

Das Cochrane Center in Kopenhagen hat im Jahr 2001 mit der Behauptung Aufmerksamkeit erregt, die Reduzierung der Sterblichkeit durch Mammographie-Screening sei nicht erwiesen. Jetzt wird von P. Götzsche, Direktor des Instituts, und M. Nielsen aufgrund neuer Studien eine Reduktion der Sterblichkeit von 15\% eingeräumt [2]. Das alte Zahlenspiel generell: Von 1000 teilnehmenden Frauen wird durch MammographieScreening nur 1 «gerettet». Rechne ich das auf die 10 Millionen anspruchsberechtigte Frauen hoch und gehe von der Teilnahmequote von $70 \%$ aus, sind es immerhin 3500 Frauen, die davon profitieren. Das neue Zahlenspiel von Götzsche/Nielsen: von 2000 Frauen wird eine gerettet.

Nicht verschwiegen werden darf die Befürchtung, dass sich durch das Mammographie-Screening über 10 Jahre bei einzelnen Frauen Brustkrebs entwickeln kann.

\section{Stand der bundesweiten Implementierung}

Laut Kooperationsgemeinschaft Mammographie ist Ende 2006 mit 53 Screening-Einheiten eine Flächendeckung von $57 \%$ erreicht. Jede Einheit versorgt etwa 100000 Frauen. Angestrebt sind insgesamt 93 Screeningeinheiten, die bis Ende 2007 etabliert sein sollen und allen anspruchsberechtigten Frauen ein Angebot zum Mammographie-Screening unterbreiten können.

\section{Ursachen für den zähen Prozess der Implementierung}

Die Etablierung des Mammographie-Screenings im deutschen Gesundheitssystem stellt eine Herausforde- 


\section{BreastCare}

rung an sich dar. Warum dauert es so lange - aus der Perspektive von betroffenen Frauen, die mit Ungeduld die Verbesserung der Versorgungsqualität bei Brustkrebs erwarten? Die entsprechenden Strukturen müssen erst geschaffen werden, die Änderungen greifen in Länderhoheiten ein, z.B. Landesdatenschutz mit Meldedatenübermittlungsverordnung, Organisation der zentralen Stellen, das 2-monatige Ausschreibungsverfahren, die 9-monatige Umsetzungsphase, Probleme mit der Haftpflichtversicherung und, und ....

\section{Probleme, die dringend gelöst werden müssen}

1. Bei positivem Befund ist die Weiterleitung der Patientin zu einer qualitätsgesicherten Therapie (z.B. Brustzentrum) nicht gewährleistet.

2. Es fehlen einheitliche Krebsregister. Eine Evaluation ist notwendig, z.B. auch bei der Frage nach den Intervallkarzinomen.

3. Wo bleiben Frauen über 70 Jahre? Die Inzidenz steigt nach dem 70. Lebensjahr noch an!

4. Profitieren Frauen der Altersgruppe 40-50 von einem Mammographie-Screening?

5. Die kurative Mammographie muss mit gleicher Qualität durchgeführt werden wie die Screening-Mammographie (durch Screening werden 30\% der Frauen erfasst).

6. Die Vorgehensweise bei zu dichtem Drüsengewebe (ACR 3 und 4) und nicht aussagefähiger Mammographie ist nicht korrekt (Nachricht an die Frau: Kein Befund).

\section{Die Perspektive}

Mammographie-Screening ist ein wichtiger Baustein sowohl in der Früherkennung als auch in der gesamten Versorgungskette bei Brustkrebs. Die Modellprojekte haben bewiesen, dass die Etablierung in Deutschland möglich ist, dass die Frauen mit dem Einladungssystem zu erreichen sind und dass Brustkrebs in einem deutlich früheren Stadium zu diagnostizieren ist, als das bisher möglich war. Damit erhöhen sich die Heilungschancen für zahlreiche Betroffene, und der Erhalt oder die Wiedererlangung von Lebensqualität sind ein Gewinn, der neben der Reduzierung der Sterblichkeit nicht unterschätzt werden darf.

Die Frauenselbsthilfe nach Krebs hat sich gemeinsam mit Frauen- und Patientenorganisationen für die Einführung des bundesweiten, flächendeckenden Mammographie-Screenings nach EU-Leitlinien eingesetzt und wird den weiteren Prozess der Implementierung und auch die Weiterentwicklung kritisch begleiten.

\section{Literatur}

1 Mammographie-Screening in Deutschland - Abschlussbericht der Modellprojekte. Kooperationsgemeinschaft Mammographie 2006. www.kooperationsgemeinschaft-mammographie.de/mammographie_screening/modellprojekte.php.

2 Götzsche PC, Nielsen M: Screening for breast cancer with mammography. Cochrane Database Syst Rev 2006;4:CD001877. DOI: 10.1002/14651858. CD001877.pub2. 